\title{
Electron Beam Infrared Nano-Ellipsometry of Individual Indium Tin Oxide
}

\section{Nanocrystals-Supplementary Information}

\author{
Agust Olafsson, ${ }^{\dagger}$ Jacob A. Busche, ${ }^{\ddagger}$ Jose J. Araujo, ${ }^{\ddagger}$ Arpan Maiti, ${ }^{\dagger}$ Juan Carlos \\ Idrobo, Daniel R. Gamelin, ${ }^{*, \ddagger}$ David J. Masiello, ${ }^{*, \ddagger}$ and Jon P. Camden*,† \\ $\dagger$ †epartment of Chemistry and Biochemistry, University of Notre Dame, Notre Dame, \\ Indiana, USA \\ $\ddagger$ Department of Chemistry, University of Washington, Seattle, Washington, USA \\ ฯ Center for Nanophase Materials Sciences, Oak Ridge National Laboratory, Oak Ridge, \\ Tennessee 37831, USA
}

E-mail: gamelin@uw.edu; masiello@uw.edu; jon.camden@nd.edu

\section{Analysis of Cubic Nanoparticle Structures}

The EEL probabilities of a series of cuboid nanoparticles are numerically calculated in order to estimate the impact of the cuboid geometries of the synthesized nanocrystals on their spectra. The lack of symmetry of the cuboids prevents the analytical derivation of their individual mode responses such that numerical calculations must suffice.

Fig. 1 shows that the primary consequence of the conversion of a spherical nanoparticle to a cubic one with large corner radii is the change of the appearance of the surface plasmon peak when the beam is placed near the particle's surface. In particular, the many independent surface plasmon modes contained within the peak become spectrally rearranged and see 


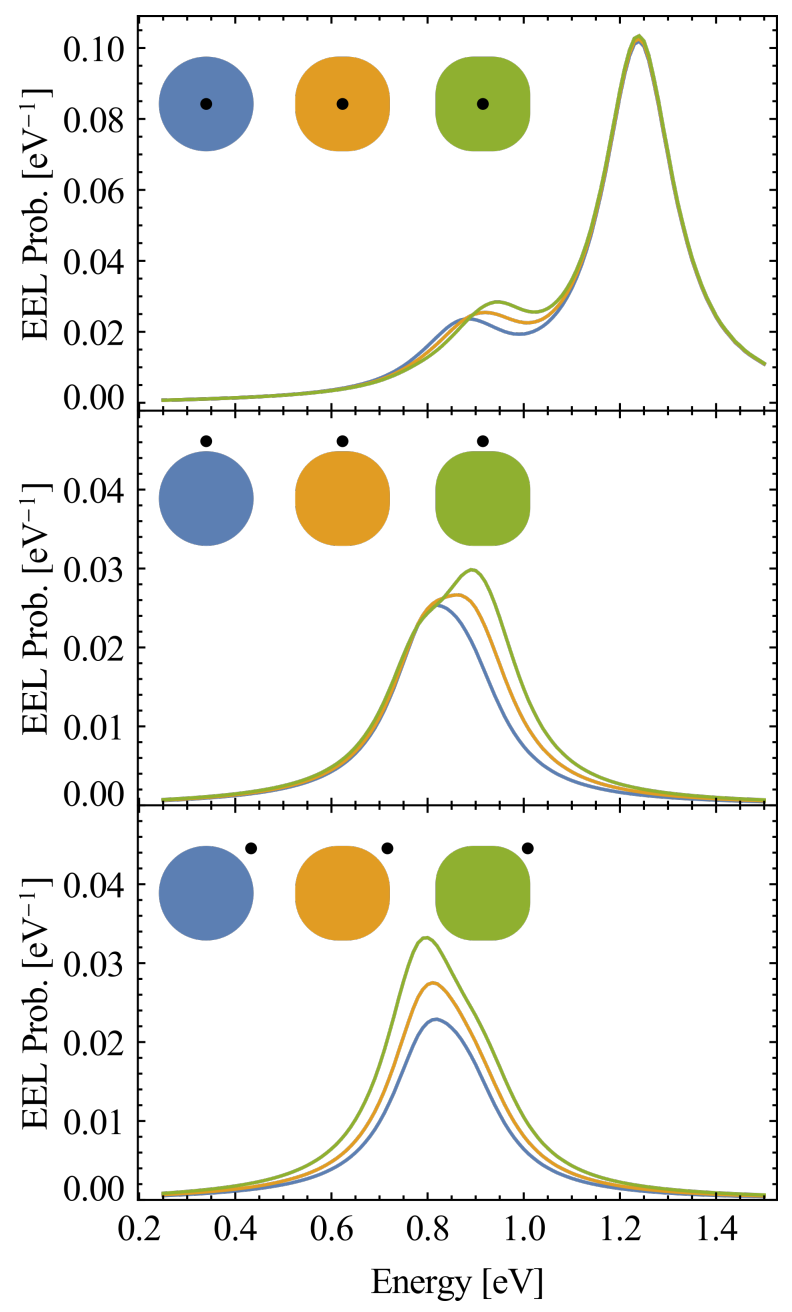

Figure 1: The EEL probability of cuboid nanoparticles with face-to-face widths $d=20 \mathrm{~nm}$ and composed of a Drude model metal with parameters equal to the extracted $10 \% \mathrm{Sn}^{4+}$ ITO parameter averages displayed in the main text. The corner radii of the cuboids are $R=d / 2$ (blue), $d / 2.2$ (gold), and $d / 2.5$ (green). The $R=d / 2$ cuboid is a sphere. Each cuboid is depicted in the three panels from above along the $z$-axis, with the upper and lower faces of each cuboid assumed to lie parallel to the $x y$-plane. The trajectory of the STEM electron lies along the $z$-axis and is depicted by the black dots in each panel. The three panels show one penetrating (top) and two aloof (middle,bottom) beam trajectories. 
their polarizabilities rise and fall such that the surface plasmon peak loses its unimodal appearance and grows a shoulder. The bulk plasmon peak is virtually unchanged throughout the evolution.

The consequence of measuring a cuboid in experiment and assuming its response to be that of a sphere is to overestimate the values of $\epsilon_{\infty}, \omega_{p}$, and $\gamma$ in the Drude dielectric model extracted from the experiment. To see this, one can first consider the penetrating cuboid spectrum: the surface plasmon feature is quite similar in cuboid and sphere spectra, except that the peak energies of the surface and bulk plasmon features are less separated.

Because the distance between the two peaks increases with decreasing $\epsilon_{\infty}$ (explicitly, $\left.\Delta \Omega \approx \omega_{p} / \sqrt{\epsilon_{\infty}}-\omega_{p} \sqrt{3 /\left[3 \epsilon_{\infty}+4\right]}\right)$, the value of $\epsilon_{\infty}$ is inferred to have an overlarge value when the cuboid is analytically assumed to be a sphere. Consequently, the value of $\omega_{p}$ is also overestimated in order to maintain the constant peak energy of the bulk feature at $\approx \omega_{p} / \sqrt{\epsilon_{\infty}}$. The value of the damping rate $\gamma$ is overestimated in aloof-beam experiments, due to the gradual splitting of the surface plasmon modes and subsequent broadening of the surface plasmon feature. The damping rate is faithfully recovered in penetrating-beam spectra as the surface plasmon feature maintains its shape, but since both aloof and penetrating spectra contribute to the extracted average value of $\gamma$, the overall effect is to overestimate it.

However, the surface spectra also provide the clearest evidence that the measured spectra are very similar to those of spheres. Even for cuboids of corner radii as large as $d / 2.2$, Fig. 1, middle panel clearly shows that the surface plasmon feature becomes markedly nonLorentzian when the aloof beam is passed near the particle's face at the midpoint between the its corners. The spectra of cuboids of smaller corner radii show surface features that are even more obviously multimodal. Such surface features are absent from the analyzed experimental data, of which a sample is shown in Fig. 2a. In each spectrum, the beam is passed across the particle's face away from any corners or protrusions (see Fig 2c), such that one would expect to see significant deformations of the surface plasmon feature were the particle to support cuboid surface plasmon modes. 
For these reasons, we estimate that the nanoparticles under investigation in this work behave like cuboids with corner radii greater than or equal to $d / 2.2$, such that the effects of the corners on the plasmon spectra are minimal. We estimate that the dielectric parameters $\omega_{p}, \gamma$, and $\epsilon_{\infty}$ are only slightly, if at all, overestimated and are within $10 \%$ of their true values.
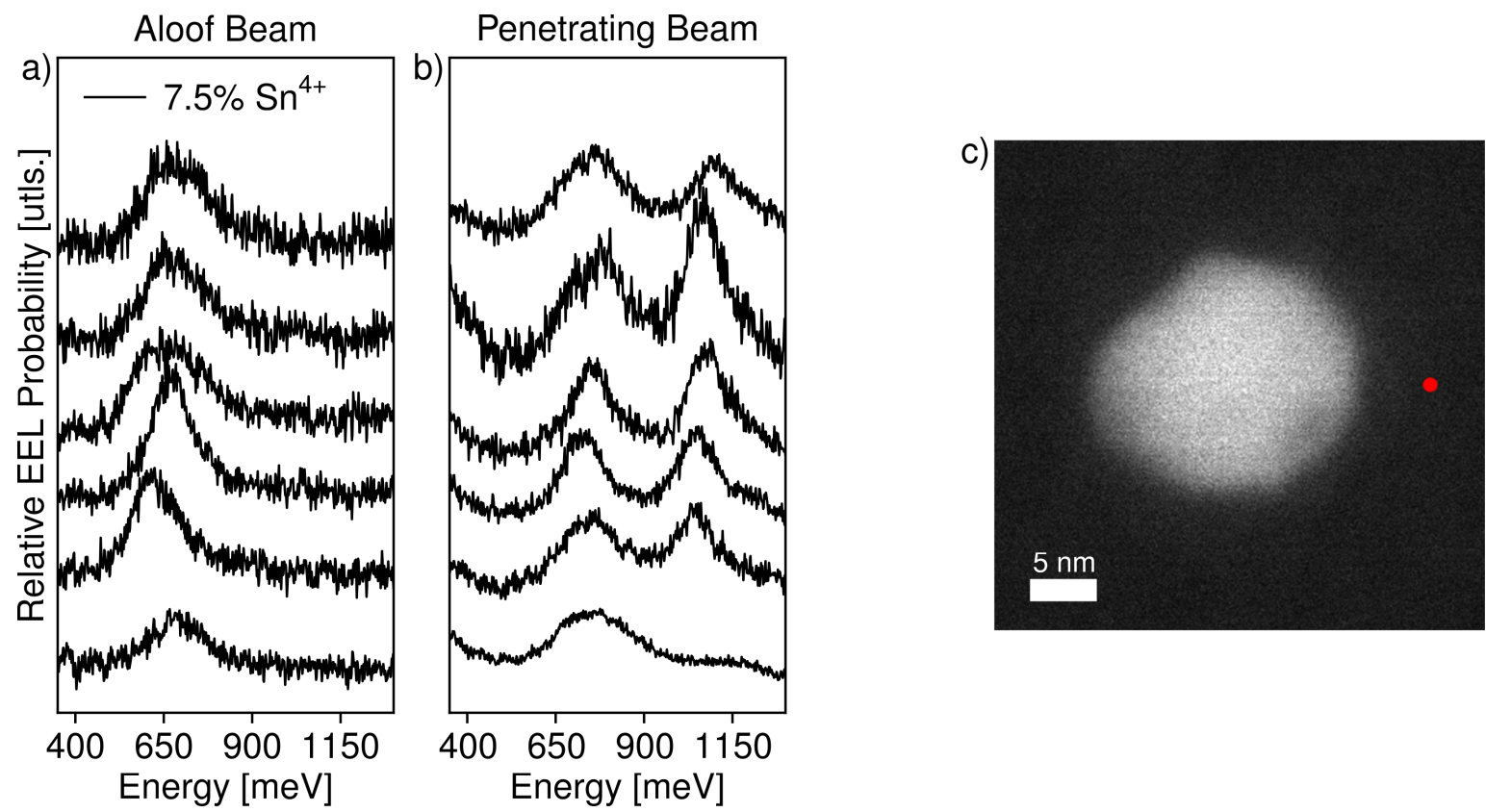

Figure 2: The aloof (a) and penetrating (b) EELS spectra for each ITO 7.5\% sample measured in this study. These plots highlight the variation in peak energy and linewidth for individual particles of the same dopant concentration, which contribute to linewidth broadening and peak energy maxima differences in ensemble measurements.

\section{Heuristic Model of the Background Signal of the EEL Probability}

To fit the loss probability $\Gamma(\omega)$ of Eq. 43 to the EEL spectra collected from experiment, the background signal must be eliminated. In the EELS low-loss energy region $(<5 \mathrm{eV})$ LSPs can overlap with losses due to Cherenkov radiation, transition radiation, inter- and intraband transitions, bulk plasmons, phonon-polaritons, and other radiative features. The position 
and intensity of these features will vary due to sample heterogeneity, but when studying LSPs they are considered background and therefore subject to removal. Additionally, the intense elastic scattering of the ZLP and its tail arise from a statistical process and can further obscure low-loss EELS data. ${ }^{1-3}$ As one might expect, the wide variety of background media in different EELS experiments leads to a corresponding variety of characteristic background signals and subtraction methods. Numerous model-based methods have been explored, including the use of power-law, reflected-tail, Gaussian, combined Gaussian and Lorentzian, Fourier and log-Fourier deconvolution, and polynomial models, as well as combinations of the above.

In order to approximate the background signal as accurately as possible, we utilized the undoped $\mathrm{In}_{2} \mathrm{O}_{3}$ NCs as a non-plasmonic "blank" to develop the model used herein. To do this, five EEL spectra from undoped $\mathrm{In}_{2} \mathrm{O}_{3}$ nanocrystals were collected with the beam passing both along the nanocrystal axis and $5 \mathrm{~nm}$ outside of its surface. The background signal was dominated by the uppermost portion of the zero loss peak (ZLP) of the electron beam below $0.3 \mathrm{eV}$ and included, depending on the beam trajectory and target dopant concentration, up to two Lorentzian-like features at $\sim 0.4 \mathrm{eV}$ and $\sim 0.8 \mathrm{eV}$. In general, the lower-energy resonance was most prominent in penetrating-beam experiments. These spectra are used to determine a characteristic range of the fitting parameters of the model

$$
\Gamma^{\text {back }}(\omega)=C+\sum_{i=1}^{3} A_{i} e^{-p_{i} \omega}+\sum_{j=1}^{2} \frac{B_{i}}{\left(\omega-\omega_{j}\right)^{2}+\gamma_{j}^{2}}
$$

This model was then numerically fit to the doped ITO samples, with the fitting parameter ranges used to determine the fitting constraints. 


\section{Analysis of Nonlinear Dielectric Effects and Beam Deflection Upon the Measured EEL Probability}

In this work we take the transverse deflection of the fast electron probe by the target to be negligible. This approximation, known as the non-recoil approximation, is known to describe the EEL signal of plasmons and other low-loss responses well when the dielectric material under investigation shows weak dependence on carrier crystal momentum and the electron detector's acceptance angle is small.

In the limit where the dielectric material of the target is strictly local, i.e., has no dependence on the momentum of the carriers contained within, the deflection of a STEM electron is sufficiently weak that the induced change in its velocity perpendicular to the direction of propagation is $\sim 10 \mathrm{~nm} / \mathrm{s}^{4}$ This behavior is similar for dielectric and metallic particles. With the axial velocity of a $60 \mathrm{keV}$ electron being $0.45 c$, the STEM electrons in this investigation are deflected at angles $\sim 10^{-18} \mathrm{rad}$, imperceptible to the detector which has an acceptance angle of $20 \mathrm{mrad}$ and can be safely set to zero in the theoretical model.

The broadening of plasmonic responses due to nonlocal effects is unimportant when examining isolated particles larger than $\sim 15 \mathrm{~nm}$, especially those with low carrier densities. As is shown in work done by Ref. 5 , nonlocal effects can significantly contribute to the plasmonic responses of coupled gold particles of this size, but do not matter much when the particles are well-isolated. Further, as can be devised from a simple hydrodynamic model of metals, ${ }^{6}$ the nonlocal response of a metal nanoparticle decreases with increasing carrier mass and decreasing carrier concentration. As compared to gold, ITO has heavier and fewer carriers. Thus, the polarization of an ITO nanoparticle is expected to have an even smaller dependence on nonlocal dielectric behaviors.

We do, however, concede that these effects are not nonexistent within ITO nanoparticles. Especially as the particles decrease in size and surface-scattering effects become exaggerated, ${ }^{7}$ the nonlocal response of carriers can significantly affect their plasmon spectra. These 
effects represent a very interesting avenue for investigation, but they can be safely neglected when modeling plasmonic spectra of isolated $\sim 20 \mathrm{~nm}$ ITO nanoparticles. As such, they lie outside the scope of this first investigation into the feasibility of nanoellipsometry.

\section{Derivation of the EEL Probability}

The dielectric model of electron energy-loss spectroscopy (EELS) begins with an electron of charge $-e$ that travels along the $-z$-direction with a speed $v$ at a lateral distance $b$ from the $z$-axis. It begins moving at a time $t \rightarrow-\infty$ and location $z \rightarrow \infty$ such that its charge distribution at a time $t$ is

$$
\rho(\mathbf{r}, t)=-\frac{e}{s} \delta(s-b) \delta(\phi-0) \delta(z+v t)
$$

with $s=\sqrt{x^{2}+y^{2}}$ the cylindrical radial coordinate and $e$ the elementary charge. The azimuthal angle of the trajectory has been chosen to be 0 for simplicity, although any angle would adequately describe the system. As this electron travels toward, through, and away from a nanosphere of radius $a$ and dielectric function $\epsilon(\omega)$ that lies centered at the origin in an otherwise isotropic universe of dielectric constant $\epsilon_{b}=1$, it interacts with the electric field $\mathbf{E}_{\text {ind }}(\mathbf{r}, t)$ that scatters from the nanosphere. The power transferred to the moving electron from this scattered field is given by the Lorentz force as

$$
P(t)=\int \mathbf{J}(\mathbf{r}, t) \cdot \mathbf{E}_{\text {ind }}(\mathbf{r}, t) \mathrm{d}^{3} \mathbf{r}
$$

wherein $\mathbf{J}$ is the electron's current and is defined via the continuity equation as

$$
\mathbf{J}(\mathbf{r}, t)=\frac{e v}{s} \delta(s-b) \delta(\phi-0) \delta(z+v t) \hat{\mathbf{z}}
$$


Moving to a picture in which we work with charges and potentials rather than currents and fields (for simplicity), the transferred power to the electron can be simply described in the quasistatic limit as

$$
\begin{aligned}
P(t) & =\int-\nabla \Phi_{\text {ind }}(\mathbf{r}, t) \cdot \mathbf{J}(\mathbf{r}, t) \mathrm{d}^{3} \mathbf{r} \\
& =\int \Phi_{\text {ind }}(\mathbf{r}, t) \nabla \cdot \mathbf{J}(\mathbf{r}, t) \mathrm{d}^{3} \mathbf{r} \\
& =-\int \Phi_{\text {ind }}(\mathbf{r}, t) \frac{\partial \rho(\mathbf{r}, t)}{\partial t} \mathrm{~d}^{3} \mathbf{r} .
\end{aligned}
$$

The total transferred energy along the electron's entire trajectory from $t \rightarrow-\infty$ to $t \rightarrow \infty$ is then

$$
\begin{aligned}
\Delta W & =\int_{-\infty}^{\infty} P(t) \mathrm{d} t \\
& =-\int_{-\infty}^{\infty} \int \Phi_{\text {ind }}(\mathbf{r}, t) \frac{\partial \rho(\mathbf{r}, t)}{\partial t} \mathrm{~d}^{3} \mathbf{r} \mathrm{d} t .
\end{aligned}
$$

The goal of the mechanical derivation of the energy transferred between the electron and the scattered fields along the electron's trajectory is to provide a concise description of the probability that the electron loses energy within a window $E+\Delta E=\hbar \omega+\hbar \Delta \omega$ to its surroundings. This probability, called the electron energy-loss (EEL) probability and given in this derivation in units of 1 /energy is defined implicitly as

$$
\Delta W=\hbar \int_{0}^{\infty} \Gamma(\omega) \hbar \omega \mathrm{d} \omega
$$

It is thus clear that any description of $\Delta W$ that will be useful must depend on the measurable quantities of the nanoparticle system as described in frequency space. The strategy for pulling $\Gamma(\omega)$ out of the integral will then involve equating the integrand above with the 
integrand of a Fourier-type integral. Letting

$$
\begin{aligned}
& \widetilde{P}(\omega)=\int_{-\infty}^{\infty} P(t) e^{i \omega t} \mathrm{~d} t \\
& P(t)=\int_{-\infty}^{\infty} \widetilde{P}(\omega) e^{-i \omega t} \frac{\mathrm{d} \omega}{2 \pi},
\end{aligned}
$$

we find that, from the convolution theorem,

$$
\widetilde{P}(\omega)=-\iint\left(i\left[\omega-\omega^{\prime}\right]\right) \widetilde{\Phi}_{\text {ind }}\left(\mathbf{r}, \omega^{\prime}\right) \tilde{\rho}\left(\mathbf{r}, \omega-\omega^{\prime}\right) \frac{\mathrm{d} \omega^{\prime}}{2 \pi} \mathrm{d}^{3} \mathbf{r}
$$

Substituting Eq. 9 into the second line of Eq. 8 and then substituting the entire right hand side of the resulting expression (of which the left hand side is $P(t)$ ) into Eq. 6, one quickly finds from an analysis of the infinite integral of an exponential function that

$$
\Delta W=\widetilde{P}(0)
$$

Therefore, because both $\Phi$ and $\rho$ are real quantities in time,

$$
\begin{aligned}
\widetilde{P}(0) & =\iint_{-\infty}^{\infty} \frac{i \omega^{\prime}}{2 \pi} \widetilde{\Phi}_{\text {ind }}\left(\mathbf{r}, \omega^{\prime}\right) \tilde{\rho}^{*}\left(\mathbf{r}, \omega^{\prime}\right) \mathrm{d} \omega^{\prime} \mathrm{d}^{3} \mathbf{r} \\
& =\iint_{0}^{\infty} \frac{i \omega^{\prime}}{2 \pi}\left[\widetilde{\Phi}_{\text {ind }}\left(\mathbf{r}, \omega^{\prime}\right) \tilde{\rho}^{*}\left(\mathbf{r}, \omega^{\prime}\right)-\text { c.c. }\right] \mathrm{d} \omega^{\prime} \mathrm{d}^{3} \mathbf{r} .
\end{aligned}
$$

Letting the dummy variable $\omega^{\prime}$ be changed as $\omega^{\prime} \rightarrow \omega$ above and equating the above expression with the integral definition of the EEL probability, one finds

$$
\Gamma(\omega)=\frac{i}{2 \pi \hbar^{2}} \int\left[\widetilde{\Phi}_{\text {ind }}(\mathbf{r}, \omega) \tilde{\rho}^{*}(\mathbf{r}, \omega)-\text { c.c. }\right] \mathrm{d}^{3} \mathbf{r} .
$$


The response potential of the system can be described with the aid of a Green function as

$$
\widetilde{\Phi}_{\text {ind }}(\mathbf{r}, \omega)=\int \widetilde{G}_{\text {ind }}\left(\mathbf{r}, \mathbf{r}^{\prime} ; \omega\right) \tilde{\rho}\left(\mathbf{r}^{\prime}, \omega\right) \mathrm{d}^{3} \mathbf{r}^{\prime}
$$

such that, if $\widetilde{G}_{\text {ind }}\left(\mathbf{r}, \mathbf{r}^{\prime} ; \omega\right)$ is known, the potential's dependence on the electron's charge distribution is clear. This form of the potential also builds in a natural symmetry to the definition of the loss probability, as

$$
\Gamma(\omega)=\frac{i}{2 \pi \hbar^{2}} \iint\left[\tilde{\rho}\left(\mathbf{r}^{\prime}, \omega\right) \widetilde{G}_{\text {ind }}\left(\mathbf{r}, \mathbf{r}^{\prime} ; \omega\right) \tilde{\rho}^{*}(\mathbf{r}, \omega)-\text { c.c. }\right] \mathrm{d}^{3} \mathbf{r} \mathrm{d}^{3} \mathbf{r}^{\prime}
$$

The quantities of interest when defining the loss probability are then the double integrals over all space, which can be shorthanded as

$$
I(\omega)=\iint \tilde{\rho}\left(\mathbf{r}^{\prime}, \omega\right) \widetilde{G}_{\text {ind }}\left(\mathbf{r}, \mathbf{r}^{\prime} ; \omega\right) \tilde{\rho}^{*}(\mathbf{r}, \omega) \mathrm{d}^{3} \mathbf{r} \mathrm{d}^{3} \mathbf{r}^{\prime}
$$

such that

$$
\begin{aligned}
\Gamma(\omega) & =\frac{i}{2 \pi \hbar^{2}}\left(I(\omega)-I^{*}(\omega)\right) \\
& =-\frac{1}{\pi \hbar^{2}} \operatorname{Im}\{I(\omega)\} .
\end{aligned}
$$

\section{Relation of the EEL probability to the nanoparticle properties}

The potential $\widetilde{\Phi}$ set up by a free charge distribution $\tilde{\rho}$ in the vicinity of dielectric objects is given by

$$
\widetilde{\Phi}(\mathbf{r}, \omega)=\int \widetilde{G}\left(\mathbf{r}, \mathbf{r}^{\prime} ; \omega\right) \tilde{\rho}\left(\mathbf{r}^{\prime}, \omega\right) \mathrm{d}^{3} \mathbf{r}^{\prime}
$$


where $\widetilde{G}$ is the Green function of the dielectric system. The response potential $\widetilde{\Phi}(\mathbf{r}, \omega)$ of the system is defined as

$$
\widetilde{\Phi}_{\text {ind }}(\mathbf{r}, \omega)=\int G_{\text {ind }}\left(\mathbf{r}, \mathbf{r}^{\prime} ; \omega\right) \tilde{\rho}\left(\mathbf{r}^{\prime}, \omega\right) \mathrm{d}^{3} \mathbf{r}^{\prime}
$$

where

$$
\widetilde{G}_{\text {ind }}\left(\mathbf{r}, \mathbf{r}^{\prime} ; \omega\right)=\widetilde{G}\left(\mathbf{r}, \mathbf{r}^{\prime} ; \omega\right)-\frac{1}{\left|\mathbf{r}-\mathbf{r}^{\prime}\right|}
$$

with $1 /\left|\mathbf{r}-\mathbf{r}^{\prime}\right|$ the Green function of free space. The free space Green function will always be present in addition to the response part, building in the direct Coulomb potential of any free charges that are present. Additionally, in general, the Green function of a system of dielectric objects with sharp boundaries takes a different form for each pair of regions that $\mathbf{r}^{\prime}$ and $\mathbf{r}$ lie in. For example, when a charge distribution lies inside a sphere of radius $a$ and dielectric $\epsilon(\omega)$ centered at the origin in an otherwise empty universe, the response Green function is given by

$$
\begin{aligned}
\widetilde{G}_{\text {ind }}^{r^{\prime}<a}\left(\mathbf{r}, \mathbf{r}^{\prime} ; \omega\right) & =\sum_{\ell=0}^{\infty} \sum_{m=-\ell}^{\ell} \frac{4 \pi}{2 \ell+1}\left(\frac{2 \ell+1}{\eta_{\ell}(\omega)}-\frac{1}{\epsilon(\omega)}\right) \frac{r^{\ell} r^{\prime \ell}}{a^{2 \ell+1}} Y_{\ell m}(\theta, \phi) Y_{\ell, m}^{*}\left(\theta^{\prime}, \phi^{\prime}\right) \Theta(a-r) \\
& +\frac{1}{\left|\mathbf{r}-\mathbf{r}^{\prime}\right|}\left(\frac{1}{\epsilon(\omega)}-1\right) \Theta(a-r) \\
& +\sum_{\ell m} \frac{4 \pi}{2 \ell+1}\left(\frac{2 \ell+1}{\eta_{\ell}(\omega)}-1\right) \frac{r^{\ell}}{r^{\prime \ell+1}} Y_{\ell m}(\theta, \phi) Y_{\ell m}^{*}\left(\theta^{\prime}, \phi^{\prime}\right) \Theta(r-a),
\end{aligned}
$$

where the Heaviside functions $\Theta$ dictate that the first two terms are nonzero when the observer is inside the sphere, and the third is nonzero when the observer is outside. Also, $\eta_{\ell}(\omega)=\ell \epsilon(\omega)+(\ell+1)$ and we have employed the identity

$$
\frac{1}{\left|\mathbf{r}-\mathbf{r}^{\prime}\right|}=\sum_{\ell m} \frac{4 \pi}{2 \ell+1} \frac{\min \left\{r, r^{\prime}\right\}^{\ell}}{\max \left\{r, r^{\prime}\right\}^{\ell+1}} Y_{\ell m}(\theta, \phi) Y_{\ell m}^{*}\left(\theta^{\prime}, \phi^{\prime}\right)
$$

in the third term. Note here that, in the second term, the subtraction of the free-space potential leaves behind a term that, although it goes like $1 /\left|\mathbf{r}-\mathbf{r}^{\prime}\right|$, actually only describes 
the polarization of the sphere's medium: in the limit $\epsilon(\omega) \rightarrow 1$, this part of the response drops to zero. When the charge distribution lies outside the sphere, the response Green function becomes

$$
\begin{aligned}
\widetilde{G}_{\text {ind }}^{r^{\prime}>a}\left(\mathbf{r}, \mathbf{r}^{\prime} ; \omega\right) & =\sum_{\ell m} \frac{4 \pi}{2 \ell+1} \frac{\ell[1-\epsilon(\omega)]}{\eta_{\ell}(\omega)} \frac{a^{2 \ell+1}}{r^{\ell+1} r^{\ell \ell+1}} Y_{\ell m}(\theta, \phi) Y_{\ell m}^{*}\left(\theta^{\prime}, \phi^{\prime}\right) \Theta(r-a) \\
& +\sum_{\ell m} \frac{4 \pi}{2 \ell+1}\left(\frac{2 \ell+1}{\eta_{\ell}(\omega)}-1\right) \frac{r^{\ell \ell}}{r^{\ell+1}} Y_{\ell m}(\theta, \phi) Y_{\ell m}^{*}\left(\theta^{\prime}, \phi^{\prime}\right) \Theta(a-r) .
\end{aligned}
$$

If a background medium had been included, a term proportional to $\left(1 / \epsilon_{b}-1\right) \times 1 /\left|\mathbf{r}-\mathbf{r}^{\prime}\right|$ would be present in the first term, but this term has been eliminated as $\epsilon_{b} \rightarrow 1$. From here, the derivation of $\Gamma(\omega)$ for a penetrating electron beam trajectory and a nanosphere target is straightforward, if nontrivial. First, with the electron treated as an unchanging point charge along its entire trajectory,

$$
\begin{aligned}
\tilde{\rho}(\mathbf{r}, \omega) & =-\frac{e}{s v} \delta(s-b) \delta(\phi-0) e^{-i \omega \frac{z}{v}} \\
& =-\frac{e}{s v} \delta(s-b) \delta(\phi-0) e^{-i \omega \frac{z}{v}} \Theta(a-r)-\frac{e}{s v} \delta(s-b) \delta(\phi-0) e^{-i \omega \frac{z}{v}} \Theta(r-a),
\end{aligned}
$$

the characteristic integral $I(\omega)$ contains four pieces:

$$
\begin{aligned}
I(\omega) & =\iint \tilde{\rho}\left(\mathbf{r}^{\prime}, \omega\right) \widetilde{G}_{\text {ind }}\left(\mathbf{r}, \mathbf{r}^{\prime} ; \omega\right) \tilde{\rho}^{*}(\mathbf{r}, \omega) \Theta\left(a-r^{\prime}\right) \Theta(a-r) \mathrm{d}^{3} \mathbf{r} \mathrm{d}^{3} \mathbf{r}^{\prime} \\
& +\iint \tilde{\rho}\left(\mathbf{r}^{\prime}, \omega\right) \widetilde{G}_{\text {ind }}\left(\mathbf{r}, \mathbf{r}^{\prime} ; \omega\right) \tilde{\rho}^{*}(\mathbf{r}, \omega) \Theta\left(a-r^{\prime}\right) \Theta(r-a) \mathrm{d}^{3} \mathbf{r} \mathrm{d}^{3} \mathbf{r}^{\prime} \\
& +\iint \tilde{\rho}\left(\mathbf{r}^{\prime}, \omega\right) \widetilde{G}_{\text {ind }}\left(\mathbf{r}, \mathbf{r}^{\prime} ; \omega\right) \tilde{\rho}^{*}(\mathbf{r}, \omega) \Theta\left(r^{\prime}-a\right) \Theta(a-r) \mathrm{d}^{3} \mathbf{r} \mathrm{d}^{3} \mathbf{r}^{\prime} \\
& +\iint \tilde{\rho}\left(\mathbf{r}^{\prime}, \omega\right) \widetilde{G}_{\text {ind }}\left(\mathbf{r}, \mathbf{r}^{\prime} ; \omega\right) \tilde{\rho}^{*}(\mathbf{r}, \omega) \Theta\left(r^{\prime}-a\right) \Theta(r-a) \mathrm{d}^{3} \mathbf{r} \mathrm{d}^{3} \mathbf{r}^{\prime} \\
& =I_{1}(\omega)+I_{2}(\omega)+I_{3}(\omega)+I_{4}(\omega) .
\end{aligned}
$$

Here we have implicitly defined the total response Green function as

$$
\widetilde{G}_{\text {ind }}\left(\mathbf{r}, \mathbf{r}^{\prime} ; \omega\right)=\widetilde{G}_{\text {ind }}^{r^{\prime}<a}\left(\mathbf{r}, \mathbf{r}^{\prime} ; \omega\right) \Theta\left(a-r^{\prime}\right)+\widetilde{G}_{\text {ind }}^{r^{\prime}>a}\left(\mathbf{r}, \mathbf{r}^{\prime} ; \omega\right) \Theta\left(r^{\prime}-a\right) .
$$


Next, we can see the $I_{2}(\omega)$ and $I_{3}(\omega)$ both contain a product of an integral over the space outside the nanoparticle and another over the space within via the action of the Heaviside functions. To simplify these terms, it is useful to first simplify the Green function terms that are nonzero in the case where $\mathbf{r}$ and $\mathbf{r}^{\prime}$ are in different regions. Letting

$$
\begin{aligned}
& f_{\ell m}^{<}(\mathbf{r})=\sqrt{\frac{4 \pi}{2 \ell+1}} \frac{r^{\ell}}{a^{\ell}} Y_{\ell m}(\theta, \phi), \\
& f_{\ell m}^{>}(\mathbf{r})=\sqrt{\frac{4 \pi}{2 \ell+1}} \frac{a^{\ell+1}}{r^{\ell+1}} Y_{\ell m}(\theta, \phi)
\end{aligned}
$$

be the mode functions that describe the spatial variation of the potential set up by the $(\ell, m)^{\text {th }}$ nanosphere mode and

$$
\begin{aligned}
\tilde{g}_{\ell}^{>}(\omega) & =\frac{\ell[\epsilon(\omega)-1]}{\eta_{\ell}(\omega)}, \\
\tilde{g}_{\ell}^{<}(\omega) & =\frac{2 \ell+1}{\eta_{\ell}(\omega)}, \\
\tilde{g}_{\ell}^{><}(\omega) & =\tilde{g}_{\ell}(\omega)-1, \\
\tilde{g}_{B}(\omega) & =\frac{1}{\epsilon(\omega)}, \\
\tilde{g}_{\infty}(\omega) & =\tilde{g}_{B}(\omega)-1,
\end{aligned}
$$

be the functions that describe the nanosphere's spectral response, we have

$$
\begin{aligned}
& \widetilde{G}_{\text {ind }}\left(\mathbf{r}, \mathbf{r}^{\prime} ; \omega\right) \Theta\left(a-r^{\prime}\right) \Theta(r-a)=\sum_{\ell m} \frac{1}{a} \tilde{g}_{\ell}^{><}(\omega) f_{\ell m}^{<}(\mathbf{r}) f_{\ell m}^{>*}\left(\mathbf{r}^{\prime}\right) \Theta\left(a-r^{\prime}\right) \Theta(r-a), \\
& \widetilde{G}_{\text {ind }}\left(\mathbf{r}, \mathbf{r}^{\prime} ; \omega\right) \Theta\left(r^{\prime}-a\right) \Theta(a-r)=\sum_{\ell m} \frac{1}{a} \tilde{g}_{\ell}^{><}(\omega) f_{\ell m}^{>}(\mathbf{r}) f_{\ell m}^{<*}\left(\mathbf{r}^{\prime}\right) \Theta\left(r^{\prime}-a\right) \Theta(a-r) .
\end{aligned}
$$

In general, the spectral responses $\tilde{g}_{\ell}$ of all three kinds describe the excitation of surfacebound phenomena (plasmons for metallic $\epsilon(\omega)$ ) and the responses $\tilde{g}_{B}$ describe the excitation 
of bulk phenomena. Using these identities,

$$
\begin{aligned}
& I_{2}(\omega)=\sum_{\ell m} \frac{\tilde{g}_{\ell}^{><}(\omega)}{a} \int_{r^{\prime}<a} \tilde{\rho}\left(\mathbf{r}^{\prime}, \omega\right) f_{\ell m}^{<*}\left(\mathbf{r}^{\prime}\right) \mathrm{d}^{3} \mathbf{r}^{\prime} \int_{r>a} \tilde{\rho}^{*}(\mathbf{r}, \omega) f_{\ell m}^{>}(\mathbf{r}) \mathrm{d}^{3} \mathbf{r} \\
& I_{3}(\omega)=\sum_{\ell m} \frac{\tilde{g}_{\ell}^{><}(\omega)}{a} \int_{r^{\prime}>a} \tilde{\rho}\left(\mathbf{r}^{\prime}, \omega\right) f_{\ell m}^{>*}\left(\mathbf{r}^{\prime}\right) \mathrm{d}^{3} \mathbf{r}^{\prime} \int_{r<a} \tilde{\rho}^{*}(\mathbf{r}, \omega) f_{\ell m}^{<}(\mathbf{r}) \mathrm{d}^{3} \mathbf{r}
\end{aligned}
$$

Additionally, with the spatial integrals shorthanded as

$$
\begin{aligned}
& A_{\ell m}^{<}(\omega)=\int_{r<a} \tilde{\rho}^{*}(\mathbf{r}, \omega) f_{\ell m}^{<}(\mathbf{r}) \mathrm{d}^{3} \mathbf{r}, \\
& A_{\ell m}^{>}(\omega)=\int_{r>a} \tilde{\rho}^{*}(\mathbf{r}, \omega) f_{\ell m}^{>}(\mathbf{r}) \mathrm{d}^{3} \mathbf{r},
\end{aligned}
$$

we have

$$
\begin{aligned}
I_{2}(\omega)+I_{3}(\omega) & =\sum_{\ell m} \frac{\tilde{g}_{\ell}^{>}(\omega)}{a}\left(A_{\ell m}^{<*}(\omega) A_{\ell m}^{>}(\omega)+A_{\ell m}^{<}(\omega) A_{\ell m}^{>*}(\omega)\right) \\
& =\sum_{\ell m} \frac{2 \tilde{g}_{\ell}^{><}(\omega)}{a} \operatorname{Re}\left\{A_{\ell m}^{<*}(\omega) A_{\ell m}^{>}(\omega)\right\} .
\end{aligned}
$$

The fourth term of $I(\omega)$ also reduces simply using the above identities to

$$
I_{4}(\omega)=\sum_{\ell m} \frac{\tilde{g}_{\ell}^{>}(\omega)}{a}\left|A_{\ell m}^{>}(\omega)\right|^{2}
$$

while the first causes more of a problem. Explicitly,

$$
I_{1}(\omega)=\sum_{\ell m} \frac{\tilde{g}_{\ell}^{<}(\omega)}{a}\left|A_{\ell m}^{<}(\omega)\right|^{2}-\sum_{\ell m} \frac{\tilde{g}_{B}(\omega)}{a}\left|A_{\ell m}^{<}(\omega)\right|^{2}+\iint_{\substack{r<a \\ r^{\prime}<a}} \tilde{\rho}\left(\mathbf{r}^{\prime}, \omega\right) \tilde{\rho}^{*}(\mathbf{r}, \omega) \frac{\tilde{g}_{\infty}(\omega)}{\left|\mathbf{r}-\mathbf{r}^{\prime}\right|} \mathrm{d}^{3} \mathbf{r} \mathrm{d}^{3} \mathbf{r}^{\prime}
$$

where the third term describes the interaction of the passing electron with the point-like screening charge set up by the polarized medium. The integral is divergent due to the behavior of the Coulombic $1 /\left|\mathbf{r}-\mathbf{r}^{\prime}\right|$ potential. To avoid this, one can replace the Coulomb 
potential with its spatial Fourier transform and neglect high-frequency terms to "smooth away" the singularity. This smoothing is consistent with a microscopic picture of an electron traveling through a crystal, wherein the electron would no longer be treated as a perfect pointlike object and would not induce an infinite polarization anywhere within the crystal.

The Fourier transform of the Coulomb potential contains a pole at $k=0$, with $\mathbf{k}$ the Fourier variable dual to $\mathbf{r}$. This pole complicates ensuing integration steps required to simplify 33. To avoid this, one can introduce an extra factor of $e^{-k_{0}\left|\mathbf{r}-\mathbf{r}^{\prime}\right|}$, with $k_{0}$ a small decay parameter, to the Coulomb potential:

$$
\frac{1}{\left|\mathbf{r}-\mathbf{r}^{\prime}\right|} \rightarrow \frac{e^{-k_{0}\left|\mathbf{r}-\mathbf{r}^{\prime}\right|}}{\left|\mathbf{r}-\mathbf{r}^{\prime}\right|}
$$

The addition of the extra factor produces a Fourier transform with a pole in $k$ away from the origin.

$$
\frac{e^{-k_{0}\left|\mathbf{r}-\mathbf{r}^{\prime}\right|}}{\left|\mathbf{r}-\mathbf{r}^{\prime}\right|}=\frac{1}{2 \pi^{2}} \int \frac{1}{k^{2}+k_{0}^{2}} e^{i \mathbf{k} \cdot\left(\mathbf{r}-\mathbf{r}^{\prime}\right)} \mathrm{d}^{3} \mathbf{k} .
$$

The introduction of the modified Coulomb potential allows one to quickly simplify the third term of Eq. 33 which, due to its resemblance to similar integrals that appear in the treatment of electron energy losses to bulk materials ${ }^{8-10}$, will be herein dubbed the "infinite-medium" integral $I_{\infty}(\omega)$. Expanding the spatial integrals and wavevector $\mathbf{k}=\mathbf{k}_{\perp}+\mathbf{k}_{z} \doteq\left(k_{\perp}, \psi, k_{z}\right)$ of $I_{\infty}$ in cylindrical coordinates and substituting the explicit forms for $\tilde{\rho}$ and $\tilde{\rho}^{*}$, one arrives at

$$
\begin{aligned}
I_{\infty}(\omega)= & \lim _{k_{0} \rightarrow \infty} \frac{e^{2} \tilde{g}_{\infty}(\omega)}{2 \pi^{2} v^{2}} \int_{-a}^{a} \int_{0}^{2 \pi} \int_{0}^{a} \int_{-a}^{a} \int_{0}^{2 \pi} \int_{0}^{a} \int \frac{1}{k^{2}+k_{0}^{2}} e^{-i \frac{\omega}{v}\left(z^{\prime}-z\right)} e^{-i k_{z}\left(z-z^{\prime}\right)} \\
& \times e^{-i k_{\perp} s \cos (\psi-\phi)} e^{-i k_{\perp} s^{\prime} \cos \left(\psi-\phi^{\prime}\right)} \delta(s-b) \delta\left(s^{\prime}-b\right) \delta(\phi-0) \delta\left(\phi^{\prime}-0\right) \\
& \times \Theta\left(a-\sqrt{s^{2}+z^{2}}\right) \Theta\left(a-\sqrt{s^{\prime 2}+z^{\prime 2}}\right) \mathrm{d}^{3} \mathbf{k} \mathrm{d} s \mathrm{~d} \phi \mathrm{d} z \mathrm{~d} s^{\prime} \mathrm{d} \phi^{\prime} \mathrm{d} z^{\prime} \\
= & \lim _{k_{0} \rightarrow \infty} \frac{e^{2} \tilde{g}_{\infty}(\omega)}{2 \pi^{2} v^{2}} \iint_{-\operatorname{Re}\left\{\sqrt{a^{2}-b^{2}}\right\}}^{\operatorname{Re}\left\{\sqrt{a^{2}-b^{2}}\right\}} \frac{1}{k_{\perp}^{2}+k_{z}^{2}+k_{0}^{2}} e^{-i\left(k_{z}-\frac{\omega}{v}\right)\left(z-z^{\prime}\right)} \mathrm{d}^{3} \mathbf{k ~ d} z \mathrm{~d} z^{\prime} .
\end{aligned}
$$


Further, with

$$
\iint_{-\operatorname{Re}\left\{\sqrt{a^{2}-b^{2}}\right\}}^{\operatorname{Re}\left\{\sqrt{a^{2}-b^{2}}\right\}} e^{-i \frac{\omega}{v}\left(z^{\prime}-z\right)} e^{-i k_{z}\left(z-z^{\prime}\right)} \mathrm{d} z \mathrm{~d} z^{\prime}=\frac{4 \sin ^{2}\left(\left[k_{z}-\frac{\omega}{v}\right] \operatorname{Re}\left\{\sqrt{a^{2}-b^{2}}\right\}\right)}{\left(k_{z}-\frac{\omega}{v}\right)^{2}},
$$

one finds that

$$
I_{\infty}(\omega)=\lim _{k_{0} \rightarrow \infty} \frac{e^{2} \tilde{g}_{\infty}(\omega)}{2 \pi^{2} v^{2}} \int \frac{4 \sin ^{2}\left(\left[k_{z}-\frac{\omega}{v}\right] \operatorname{Re}\left\{\sqrt{a^{2}-b^{2}}\right\}\right)}{\left(k_{z}-\frac{\omega}{v}\right)^{2}\left(k_{\perp}^{2}+k_{z}^{2}+k_{0}^{2}\right)} \mathrm{d}^{3} \mathbf{k} .
$$

The $k_{z}$-integral of Eq. 38 can be evaluated analytically with the aid of the symbolic integration library of Mathematica 12.1. Explicitly,

$$
\begin{aligned}
I_{\infty}(\omega)= & \lim _{k_{0} \rightarrow \infty} \frac{e^{2} \tilde{g}_{\infty}(\omega)}{\pi v^{2}} \int_{0}^{\infty} k_{\perp} \mathrm{d} k_{\perp}\left(\frac{4 \pi \operatorname{Re}\left\{\sqrt{a^{2}-b^{2}}\right\}}{\frac{\omega^{2}}{v^{2}}+k_{\perp}^{2}+k_{0}^{2}}\right. \\
+ & \frac{2 \pi}{\sqrt{k_{\perp}^{2}+k_{0}^{2}}} \operatorname{Re}\left\{\frac{1}{\left(\frac{\omega}{v}-i \sqrt{k_{\perp}^{2}+k_{0}^{2}}\right)^{2}}\right. \\
& \times\left(1-\cos \left[2\left(\frac{\omega}{v}-i \sqrt{k_{\perp}^{2}+k_{0}^{2}}\right) \operatorname{Re}\left\{\sqrt{a^{2}-b^{2}}\right\}\right]\right. \\
& \left.\left.+i \sin \left[2\left(\frac{\omega}{v}-i \sqrt{k_{\perp}^{2}+k_{0}^{2}}\right) \operatorname{Re}\left\{\sqrt{a^{2}-b^{2}}\right\}\right]\right)\right\},
\end{aligned}
$$

where the $\psi$-integral has simply produced a factor of $2 \pi$ as the integrand of Eq. 38 is independent of $\psi$. Taking the integral above requires the introduction of a cutoff wavevector for the $k_{\perp}$ integral such that the highest spatial frequency components of the Coulomb potential are removed. One can see that the above integral diverges without this approximation. Therefore, in the limit $k_{0} \rightarrow 0$ and with the integration bounds of $k_{\perp}$ limited to $\left[0, k_{c}\right]$, we 
have

$$
\begin{aligned}
I_{\infty}(\omega) & \approx \frac{e^{2} \tilde{g}_{\infty}(\omega)}{\pi v^{2}}\left(2 \pi \sqrt{a^{2}-b^{2}} \ln \left[1+\frac{k_{c}^{2} v^{2}}{\omega^{2}}\right]+\frac{2 \pi k_{c}}{k_{c}^{2}+(\omega / v)^{2}}-\frac{2 \pi \sin \left[2 \sqrt{a^{2}-b^{2}}(\omega / v)\right]}{\omega / v}+\right. \\
& \frac{2 \pi}{k_{c}^{2}+(\omega / v)^{2}} e^{-2 k_{c} \sqrt{a^{2}-b^{2}}}\left[\frac{\omega}{v} \sin \left(2 \sqrt{a^{2}-b^{2}}[\omega / v]\right)-k_{c} \cos \left(2 \sqrt{a^{2}-b^{2}}[\omega / v]\right)\right]+ \\
& \left.2 \pi \sqrt{a^{2}-b^{2}}\left[\pi+2 \operatorname{Re}\left\{\Gamma\left(0,2\left[k_{c}+i(\omega / v)\right] \sqrt{a^{2}-b^{2}}\right)\right\}\right]\right) .
\end{aligned}
$$

Here, $\Gamma(n, x)$ is the upper incomplete Euler gamma function and should not be confused with a loss probability. For physical values of the parameters $\left(\omega \sim 10^{15} \mathrm{~Hz}, v \sim 10^{10} \mathrm{~cm} / \mathrm{s}\right.$, $\left.a, b \sim 10^{-6} \mathrm{~cm}\right)$, the result of Eq. 40 is $\sim 10^{-4} \mathrm{~cm}^{2} \mathrm{~g}$ and becomes increasingly dominated by the first term as $k_{c}$ becomes much greater than $\sim 10^{6} \mathrm{~cm}^{-1}$. In particular, the second and fourth terms decrease straightforwardly with increasing $k_{c}$, while the fifth is generally monotonically decreasing in $k_{c}$ for $k_{c}>\omega / v$ and the third provides a small $\left(\sim 10^{-6} \mathrm{~cm}^{2} \mathrm{~g}\right)$ negative correction to the total integral.

Closely related strategies to simplify $I_{\infty}(\omega)$ exist in Refs. ${ }^{8,9}$. These models treat a simpler form of the infinite-medium integral of Eq. 36 that is appropriate for an electron moving through an infinite, uniform material. First calculating an EEL probability per unit path length for such an electron, it is shown that one can then integrate along the electron's path through a sphere to approximate the infinite-medium EEL probability. At the level of the integral $I_{\infty}(\omega)$, this is performed by allowing the integration bounds on $z^{\prime}$ to go to infinity, such that

$$
I_{\infty}(\omega) \rightarrow \frac{e^{2} \tilde{g}_{\infty}(\omega)}{2 \pi^{2} v^{2}} \int_{-\infty}^{\infty} \int_{-\operatorname{Re}\left\{\sqrt{a^{2}-b^{2}}\right\}}^{\operatorname{Re}\left\{\sqrt{a^{2}-b^{2}}\right\}} \int \frac{k_{\perp}}{k^{2}} e^{-i\left(k_{z}-\frac{\omega}{v}\right)\left(z-z^{\prime}\right)} \mathrm{d}^{3} \mathbf{k} \mathrm{d} z \mathrm{~d} z^{\prime}
$$


Using the same cutoff wavenumber $k_{c}$ to regularize the Coulomb potential, this delivers

$$
I_{\infty}(\omega) \approx \frac{2 e^{2} \tilde{h}_{2}(\omega) \sqrt{a^{2}-b^{2}}}{v^{2}} \ln \left(1+\frac{k_{c}^{2} v^{2}}{\omega^{2}}\right)
$$

which is simply the first term of Eq. 40 and is appropriate for large enough $k_{c}$. Comparison with Ref. ${ }^{9}$ can be made by letting $\phi_{\text {out }}=\tan ^{-1}\left(\hbar k_{c} / m_{e} v\right) \approx \hbar k_{c} / m_{e} v$ be the detector's maximum collection half-angle.

Because the dielectric model without momentum dispersion neglects many important phenomena that affect the bulk EEL probability ${ }^{8,9}$, the simpler expression for $I_{\infty}$ found in Eq. 42 is used in this work. The relative magnitudes of the bulk and surface plasmon EEL features are unimportant to the inference of the Drude parameters, so $k_{c}$ is fit as necessary but is not examined for its physical significance. A detailed determination of the contributions of the latter terms of Eq. 40 to the bulk EEL probability, as well of terms that arise due to the inclusion of physics beyond the dielectric model, is beyond the scope of this investigation.

Finally, one can see that the imaginary parts of $I_{1}, I_{2}, I_{3}, I_{4}$, and $I_{\infty}$ are all determined solely by the imaginary parts of the response functions $\tilde{g}_{i}$ :

$$
\begin{aligned}
\Gamma(\omega)=- & \frac{1}{a \pi \hbar^{2}} \sum_{\ell m}\left(\left|A_{\ell m}^{<}(\omega)\right|^{2} \operatorname{Im}\left\{\tilde{g}_{\ell}^{<}(\omega)\right\}+\left|A_{\ell m}^{>}(\omega)\right|^{2} \operatorname{Im}\left\{\tilde{g}_{\ell}^{>}(\omega)\right\}\right) \\
& +2 \operatorname{Re}\left\{A_{\ell m}^{<}(\omega) A_{\ell m}^{>}(\omega)\right\} \operatorname{Im}\left\{\tilde{g}_{\ell}^{><}(\omega)\right\} \\
& +\frac{1}{a \pi \hbar^{2}} \sum_{\ell m}\left|A_{\ell m}^{<}(\omega)\right|^{2} \operatorname{Im}\left\{\tilde{g}_{B}(\omega)\right\}-\frac{1}{\pi \hbar^{2}} \operatorname{Im}\left\{I_{\infty}(\omega)\right\} \\
= & \Gamma^{\operatorname{surf}}(\omega)+\Gamma^{\text {geo }}(\omega)+\Gamma^{\infty}(\omega) .
\end{aligned}
$$




\section{Dependence upon Drude-Model Parameters of the Mixing Angle between an LSP and a Nearby Reso-} nance

The instantaneous interaction energy between a localized surface plasmon (LSP) mode of multipole order $\ell, m$ and a neighboring resonance is given by

$$
U(t)=\int \Phi_{\ell m}(\mathbf{r}, t) \rho_{n}(\mathbf{r}, t) \mathrm{d} \mathbf{r}
$$

where $\Phi_{\ell m}$ is the potential set up by the oscillating LSP mode and $\rho_{n}$ is the charge density of the resonance. If the resonance is taken to be an oscillating dipole centered at a location $\mathbf{r}_{0}$, then

$$
\rho_{n}(\mathbf{r}, t)=-\mathbf{p}(t) \cdot \nabla \delta\left(\mathbf{r}-\mathbf{r}_{0}\right)
$$

where $\mathbf{p}(t)$ is the resonance's time-dependent dipole moment. Further, with the LSP's charge distribution taken to be a surface charge density given by

$$
\rho_{\ell m}(\mathbf{r}, t)=\frac{q_{\ell m}(t)}{a^{\ell+2}} Y_{\ell m}(\theta, \phi) \delta(r-a)
$$

that is proportional to its multipole moment $q_{\ell m}(t)$ (given here in the spherical coordinate convention for convenience), the interaction energy can be redefined using Coulomb's law to give

$$
\begin{aligned}
U(t) & =\iint \frac{\rho_{\ell m}\left(\mathbf{r}^{\prime}, t\right) \rho_{n}(\mathbf{r}, t)}{\left|\mathbf{r}-\mathbf{r}^{\prime}\right|} \mathrm{d}^{3} \mathbf{r} \mathrm{d}^{3} \mathbf{r}^{\prime} \\
& =-\frac{q_{\ell m}(t) \mathbf{p}(t)}{a^{\ell+2}} \cdot \int \frac{\mathbf{r}_{0}-\mathbf{r}^{\prime}}{\left|\mathbf{r}_{0}-\mathbf{r}^{\prime}\right|^{3}} Y_{\ell m}\left(\theta^{\prime}, \phi^{\prime}\right) \delta\left(r^{\prime}-a\right) \mathrm{d}^{3} \mathbf{r}^{\prime},
\end{aligned}
$$

where the second line was derived using integration by parts. Further, as the time-dependent multipole moments encode the motion of two massive oscillators, it is sensible to assign to 
each of them a coordinate $Q$, such that $e Q_{\ell m}(t)=q_{\ell m}(t)$ and $e Q_{n}(t) \hat{\mathbf{p}}=\mathbf{p}(t)$. Therefore, letting

$$
\sigma=\frac{e^{2}}{a^{\ell+2}} \hat{\mathbf{p}} \cdot \int \frac{\mathbf{r}_{0}-\mathbf{r}^{\prime}}{\left|\mathbf{r}_{0}-\mathbf{r}^{\prime}\right|^{3}} Y_{\ell m}\left(\theta^{\prime}, \phi^{\prime}\right) \delta\left(r^{\prime}-a\right) \mathrm{d}^{3} \mathbf{r}^{\prime}
$$

be the coupling strength between the two resonances, one is left with the simple equations of motion of the coupled system

$$
\begin{gathered}
m_{\ell} \ddot{Q}_{\ell m}(t)+m_{\ell} \gamma \dot{Q}_{\ell m}(t)+m_{\ell} \omega_{\ell}^{2} Q_{\ell m}(t)-\sigma Q_{\ell m}(t) Q_{n}(t)=F_{\ell m}(t), \\
m_{n} \ddot{Q}_{n}(t)+m_{n} \gamma \dot{Q}_{n}(t)+m_{n} \omega_{n}^{2} Q_{n}(t)-\sigma Q_{n}(t) Q_{\ell m}(t)=F_{n}(t),
\end{gathered}
$$

where the definitions of the effective masses $m_{i}$, natural frequencies $\omega_{i}$, and external forces $F_{i}(t)$ of each oscillator are implied with the knowledge that $\omega_{\ell m}=\omega_{\ell}$ and $m_{\ell m}=m_{\ell}$. The above equations can be decoupled in the limit where the damping rate is small to give

$$
\begin{aligned}
& \ddot{Q}_{+}(t)+\Omega_{+}^{2} Q_{+}(t)=\frac{F_{+}(t)}{m_{\ell m}}, \\
& \ddot{Q}_{-}(t)+\Omega_{-}^{2} Q_{-}(t)=\frac{F_{-}(t)}{m_{n}},
\end{aligned}
$$

where $Q_{ \pm}, \Omega_{ \pm}$, and $F_{ \pm}$are the normal mode coordinates, resonance frequencies, and forces of the system. We will define only the resonance frequencies here, which take the form

$$
\Omega_{ \pm}^{2}=\omega_{\ell m}^{2} \cos ^{2}(\beta)+\omega_{n}^{2} \sin ^{2}(\beta) \mp \frac{\sigma}{\sqrt{m_{\ell} m_{n}}} \sin (2 \beta)
$$

where $\beta$ is the mixing angle defined by

$$
\beta=\frac{1}{2} \tan ^{-1}\left\{\frac{2 \sigma}{\sqrt{m_{\ell} m_{n}}\left(\omega_{\ell}^{2}-\omega_{n}^{2}\right)}\right\} .
$$

The LSP masses are defined by convention when the generalized coordinate displacements are related to the time-dynamics of the external forces. They cannot be uniquely determined, however the choice of LSP masses cannot affect the time-evolution of any observable 
quantities. One convenient convention for $m_{\ell}$ is chosen in Ref. ${ }^{11}$, wherein

$$
m_{\ell}=\frac{2 \pi e^{2}\left(\ell \epsilon_{\infty}+[\ell+1]\right)}{(2 \ell+1) \omega_{\ell}^{2} a^{3}}
$$

such that, with $\omega_{\ell}=\omega_{p} \sqrt{\ell /\left(\ell \epsilon_{\infty}+[\ell+1]\right)}$, the LSP effective mass scales with the material parameters as $m_{\ell} \sim\left(\ell \epsilon_{\infty}+[\ell+1]\right)^{2} / \omega_{p}^{2}$. Assuming the coupled system is maintained at a fixed detuning $\Delta^{2}=\omega_{\ell}^{2}-\omega_{n}^{2}$, coupling strength $\sigma$, nanosphere radius $a$, and resonance mass $m_{n}$, it is readily apparent that the mixing angle then becomes

$$
\beta=\frac{1}{2} \tan ^{-1}\left\{\frac{2 \sigma \sqrt{\ell(2 \ell+1) a^{3}}}{e \sqrt{2 \pi m_{n}} \Delta^{2}} \frac{\omega_{p}}{\ell \epsilon_{\infty}+(\ell+1)}\right\},
$$

such that the leftmost fraction in the curly braces of Eq. 54 is a constant. Thus, maximal mixing always occurs a large $\omega_{p}$ and small $\epsilon_{\infty}$.

\section{References}

(1) Wu, Y.; Li, G.; Camden, J. P. Probing nanoparticle plasmons with electron energy loss spectroscopy. Chemical Reviews 2017, 118, 2994-3031.

(2) Egerton, R. F. Electron energy-loss spectroscopy in the TEM. Reports on Progress in Physics 2008, 72, 016502.

(3) Hachtel, J. A.; Lupini, A. R.; Idrobo, J. C. Exploring the capabilities of monochromated electron energy loss spectroscopy in the infrared regime. Scientific Reports 2018, 8, 110.

(4) García de Abajo, F. J. Momentum transfer to small particles by passing electron beams. Physical Review B 2004, 70, 115422.

(5) García de Abajo, F. J. Nonlocal effects in the plasmons of strongly interacting nanopar- 
ticles, dimers, and waveguides. The Journal of Physical Chemistry C 2004, 112, 1798217987.

(6) Ródriguez-Suné, L.; Scalora, M.; Johnson, A. S.; Cojocaru, C.; Akozbek, N.; Coppens, Z. J.; Perez-Salinas, D.; Wall, S.; Trull, J. Study of second and third harmonic generation from an indium tin oxide monolayer: Influence of nonlocal effects and hot electrons. APL Photonics 2020, 5, 010801.

(7) Apell, P.; Penn, D. R. Optical Properties of Small Metal Spheres: Surface Effects. Physical Review Letters 1983, 50, 1316-1319.

(8) Ritchie, R. H. Plasma losses by fast electrons in thin films. Physical Review 1957, 106, 874.

(9) García de Abajo, F. J. Optical excitations in electron microscopy. Reviews of Modern Physics 2010, 82, 209.

(10) Pines, D.; Bohm, D. A Collective Description of Electron Interactions: II. Collective vs Individual Particle Aspects of the Interactions. Physical Review 1952, 85, 338.

(11) Cherqui, C.; Thakkar, N.; Li, G.; Camden, J. P.; Masiello, D. J. Characterizing Localized Surface Plasmons Using Electron Energy-Loss Spectroscopy. Annual Review of Physical Chemistry 2016, 67, 331-357. 